\title{
Characterizing Soil Preferential Flow Using Iodine-Starch Staining Experiments and the Active Region Model
}

\author{
Feng Shenga, Kang Wang ${ }^{b}$, Renduo Zhanga, and Hui-Hai Liuc \\ aSchool of Environmental Science and Engineering, Sun Yat-Sen \\ (Zhongshan) University, Guangzhou 510275, China
}

bState Key Lab. of Water Resources and Hydropower Engineering Science, Wuhan University, Wuhan 430072, China

cEarth Sciences Division, Lawrence Berkeley National Laboratory, Berkeley, CA, USA 


\begin{abstract}
Thirteen iodine-starch staining experiments with different boundary conditions and measurement scales were conducted at two sites to study preferential flow processes in natural unsaturated soils. Digital imaging analyses were implemented to obtain the corresponding preferential flow patterns. The test results are used to evaluate a recently proposed active region model in terms of its usefulness and robustness for characterizing unsaturated flow processes at field scale.
\end{abstract}

Test results provide useful insights into flow patterns in unsaturated soils. They show that flow pattern depends on the top boundary condition. As the total infiltrating-water depth increased form $20 \mathrm{~mm}$ to $80 \mathrm{~mm}$ for the $100 \times 100 \mathrm{~cm}^{2}$ plots, the corresponding flow pattern changed from few preferential flow paths associated with a relatively small degree of stained coverage and a small infiltration depth, to a pattern characterized by a higher stained coverage and a larger infiltration depth, and to (finally) a relatively homogeneous flow pattern with few unstained area and a much larger infiltration depth. Test results also show that the preferential flow pattern became generally more heterogeneous and complex for a larger measurement scale (or size of infiltration plot). These observations support the general idea behind the 
active region model that preferential flow pattern in unsaturated soils are dynamic and depend on water flow conditions.

Further analyses of the test results indicate that the active-region model is able to capture the major features of the observed flow pattern at the scale of interest, and the determined parameter values do not significantly depend on the test conditions (initial water content and total amount of infiltrating water) for a given test site. This supports the validity of the active region model that considers that parameter to be a property of the corresponding unsaturated soil. Results also show that some intrinsic relation seems to exist between active-fracture-model parameter and a random-cascade-model parameter. (The latter model is also developed based on the existence of the fractal flow pattern in unsaturated soils.) Furthermore, our test results demonstrate that the active-region-model parameter is not scale-dependent for a range of scales under consideration. Although further studies are needed to confirm this finding, it seems to be consistent with a consideration that some fractal parameters (e.g., fractal dimension) are universal for a large range of scales.

Key Words: Active region model; fractal; dye infiltration experiment; heterogeneous water flow; random cascade model 


\section{INTRODUCTION}

Spatial variability of the soil properties at field scale make it difficult to describe unsaturated soil-water flow and transport. One important and common phenomenon in unsaturated soil is preferential flow, which is difficult to capture by using traditional continuum methods (Doughty, 1999). Even for homogeneous and structureless soil, due to high nonlinearity of unsaturated flow processes, an infiltration front can become unstable and split into fingers (Glass et al., 1988, Liu et al., 1998; Pruess et al., 1999; de Rooij, 2000).

A variety of approaches have been developed to deal with preferential flow. These approaches mainly fall into two categories: continuum and discrete (Liu et al., 2005). Several commonly used continuum-approach-based models, from the relative simple single-porosity model to more complex physically based dual-porosity, dual-permeability, and multi-region type models, were reviewed by Simunek et al., (2003) in a study of macropore flow and transport in the vadose zone. Though they are relatively simple and straightforward to implement, traditional continuum approaches are incapable of characterizing the preferential flow paths caused by fingering and the spatial variability of soil properties (Liu et al., 2005). In contrary, discrete approaches, such as the diffusion-limited aggregation model (Persson et al., 2001) and the random cascade model (Olsson et al., 2002), have been successfully 
used to represent field observations. The success of discrete approaches in modeling preferential flow mainly relies on their capability for generating fractal and multifractal patterns. However, these discrete approaches are limited for large-scale applications (Liu et al., 2003) and the substantial physical mechanisms underlying these approaches are still absent.

To properly characterize heterogeneous water flow processes in the soil, and to benefit from the combined advantages of continuum and discrete approaches, Liu et al. (2005) incorporated fractal theory within the traditional continuum approaches and developed the active region model (ARM). While the previous studies of the field observations (van Dam et al., 1990; Larsson et al., 1999; Ohrstrom et al., 2002) supported this theory, an in-depth evaluation on the ARM has not yet been conducted.

Similar to the dual-domain model (van Dam et al., 1990, 1996; Larsson et al., 1999), the ARM divides the whole flow region into an active (mobile) and an inactive (immobile) region. Water flow occurs in the active region exclusively, allowing the inactive region to be simply bypassed. The major difference between the ARM and the dual-domain model is that, in the ARM, the active region is fractal and changeable, and the relative portion of the active region is expressed as a power function of the water saturation within the flow region. As the discrete approaches use the fractal dimension to characterize the heterogeneous flow patterns, the ARM parameter 
(denoted by $\gamma$ ) is used to describe the preferential flow patterns. However, most of the discrete models described flow stained patterns without taking soil saturation within the flow region into consideration. For instance, the parameter $(\sigma)$, used in an random cascade model with lognormal distribution of infiltration depth (Wang et al., 2006), increases as flow patterns become more heterogeneous. Whereas the consistency between field observations and proposed models support both ARM and the random cascade model for characterizing preferential flow paths, it is necessary to investigate the relations between $\gamma$ and other fractal parameters used in the discrete approaches to describe flow patterns to better understand flow patterns in soil and some of the physical insights provided by the active region model.

To evaluate the ARM and relations between $\gamma$ and field observations, it is needed to visualize the flow region first in order to determine the active flow region from results of digital imaging analysis (Morris and Mooney; 2004). One also needs the water saturation distribution (within the flow regions) determined by soil sampling. For flow path visualization, various tracers such as Brilliant Blue (e.g., Yasuda et al., 2001; Morris and Mooney, 2004; Ohrström et al., 2004), or pH indications of alkaline infiltration water (Wang et al., 2002, 2003) can be suitable, depending on site properties. Ketelsen and Meyer-Windel (1999) found that Brilliant Blue is adsorbed to clay minerals and the organic carbon seems to inhibit the adsorption by masking clay 
adsorption points. Soil with high clay and low organic carbon content tends to adsorb more dyes than others (Kasteelet al., 2002). Other factors, such as $\mathrm{pH}$ and calcium content (Flury and Fluhler, 1995), also affect adsorption. In the study by Ohrstrom et al. (2004), combined tracers of Brilliant Blue and bromide were used, with observed dye patterns and measurement of the bromide concentration showing large heterogeneity. The dye did not move through the total soil matrix, but rather stayed within preferential flow paths. Iodine-starch staining was proposed as an alternative method to investigate flow pathways (Hendrikx, et al, 1988, van Ommen et al., 1989, Flury and Fluhler, 1995, Hangen et al., 2004). In addition to the wonderful advantages of no toxicity, distinct visibility, and similar transport properties to water (Flury and Fluhler, 1994), high water solubility and anionic properties provide the iodine ion with high mobility and low adsorption, and make the iodine ion as an ideal dye tracer, especially in heavy clay soil. Based on the above considerations, the iodine ion was used in this study.

The major objective of this work is to evaluate the ARM with observed soil-water flow patterns and the associated measurements collected from our field tests. The study also tends to develop further insight into underlying relations between flow patterns and the ARM parameter. Specifically, we will (1) study preferential flow patterns for a loam and a clay soil using iodine-starch staining, (2) characterize these 
patterns for various boundary conditions and measurement scales, and (3) determine relationships between the ARM parameter and field test conditions. In addition, we also discuss relations between fitted values for the ARM parameter and those for a random-cascade-model parameter.

\section{METHODS AND MATERIALS}

\subsection{Field experiments}

Dye infiltration experiments were conducted at two different field sites under various hydraulic conditions, from June 2006 to Oct. 2007. One site, denoted here as Site I, was located in the Irrigation and Drainage Station of Wuhan University, China. Before the experiment, the site was used for vegetation production. The soil texture was loam with visually uniform profiles. The second site, denoted as Site II, was located at the foot of Mountain LuoJia, China. At this site, obvious macropores were observed in the top $40 \mathrm{~cm}$ layer. The second layer (below $40 \mathrm{~cm}$ ) consisted of unstructured clay. Before the experiment, the top $40 \mathrm{~cm}$ of soil was removed. Soil samples were collected at each site at the depths of 0-10, 10-20, 20-50 and 50-100 cm to investigate soil properties, including the soil texture, bulk density, porosity, and saturated hydraulic conductivity, which are summarized in Table 1. The soil water retention function was estimated from soil texture; the residual water contents are 
0.07 and 0.12 for the loam and clay soils, respectively.

Before the experiments, an iodide indication solution was prepared with a concentration of $50 \mathrm{~g} \mathrm{~L}^{-1}$, as suggested by $\mathrm{Lu}$ and $\mathrm{Wu}$ (2003). A $50 \mathrm{~g}$ water soluble linear starch was mixed with $0.05 \mathrm{~L}$ distilled water, then added to $0.8 \mathrm{~L}$ boiling water, keeping stirring until the solution become clear. After that, cooled the solution at room temperature. To make the iodide entirely oxidized (from $\mathrm{I}^{-}$to $\mathrm{I}_{2}$ ) in the atmosphere, $40.40 \mathrm{~g} \mathrm{Fe}\left(\mathrm{NO}_{3}\right)_{3} \cdot 9 \mathrm{H}_{2} \mathrm{O}$ was added to the starch solution. Stirred the solution until completely dissolved, then titrated the volume to $1.0 \mathrm{~L}$.

Under field conditions, $10 \mathrm{~mL}$ Brilliant Blue dye solution with a concentration of $4 \mathrm{~g} / \mathrm{L}$ and $10 \mathrm{~mL}$ iodide solution with a concentration of $20 \mathrm{~g} / \mathrm{L}$ were applied to show the difference. The excavated dye profiles indicated that the mean transport distances of Brilliant Blue $(5.4 \mathrm{~cm}$ and $8.5 \mathrm{~cm}$ at Site I and Site II, respectively) were significantly less than those of iodide $(7.4 \mathrm{~cm}$ and $12.5 \mathrm{~cm}$ at Site I and Site II, respectively). The high adsorption of Brilliant Blue by the soil particles made those differences.

A total of 13 dye infiltration experiments were conducted at the two sites, with 7 conducted at Site I and 6 conducted at Site II. The infiltration conditions for each plot are shown in Table 2. The experimental design is schematically shown in Fig. 1 (a, b). Two rectangular frames were concentrically embedded into each plot. The outer frame 
is larger than the inner frame by $1 \mathrm{~m}$ in both length and width. Before infiltration, each plot was leveled to ensure a uniform surface boundary condition. To avoid interference, the plots were placed at least $2 \mathrm{~m}$ apart from each other. The inner plot and outer area were covered with plastic film. At the beginning of the dye experiments, the inner plots were prepared by ponding potassium iodide solution (20 $\mathrm{g} / \mathrm{L}$ ) on the top of the plastic film, with the outer border area flooded with the same depth of fresh water. When the experiment started, the plastic film was removed immediately, thus creating an almost instantaneous ponding infiltration in both inner and border plots. Then the plots were covered to prevent evaporation and left for 12 hours to complete the infiltration process (Yasuda et al., 2001; Wang et al., 2006).

Twelve hours after tracer applied, the flow region visualization was started. In the plots of Site I, the soil was removed layer by layer (Fig 1 (a)) at a vertical interval of 1 to $5 \mathrm{~cm}$ from top to bottom. In the plots of Site II, a trench was manually dug to a depth of $1.5 \mathrm{~m}, 1.0 \mathrm{~m}$ apart from the experiment plots, one day before the dye solution was applied. After infiltration, vertical soil profiles were excavated across the plots at a horizontal interval of $5 \mathrm{~cm}$. After each layer or profile was exposed, the surface was leveled and cleaned with a brush to remove soil particles resulted from digging. A pressurized sprayer that releases a continuous fine mist was used to apply the indication solution onto the surface as uniformly as possible. 
Under atmospheric conditions, $75 \%$ to $85 \%$ of the total amount of ionic iodide is converted into molecular iodine (Behrens, 1982). The iodine molecules enter the tube-like starch constituent amylase, being stabilized and displaying a distinct purple color (van Ommen et al., 1988). This color reaction mainly occurs at the beginning 10 minutes after the indication solution was sprayed. Staining patterns were recorded during day time using a CCD digital camera, with white sheet used to diffuse the light and to avoid direction radiation (Forrer et al., 2000). Each pixel in the color images was classified as "stained" or "unstained" based on its red, green, or blue values (Forrer et al., 2000). Each pixel represents $1 \mathrm{~mm}^{2}$ of the original soil profile.

After visualization, soil samples were collected within the stained regions and the unstained regions to investigate the soil water content distributions. Specially, for the plots at Site II, soil samples were collected at depths of 0, 10, 2030 40, 50, 60, 70 and $80 \mathrm{~cm}$ in both the stained and unstained regions.

\subsection{ARM and determination of its parameter values}

While the details of the ARM can be found in Liu et al. (2005), we give a brief introduction to the ARM concepts and the relevant parameters herein. In the traditional continuum approaches, the whole flow region is considered to be conductive, the flow and transport occurs in the whole flow region, and all the 
parameters used in the governing equations are related to the whole flow region. The main idea behind the ARM is that flow domain can be divided into active and inactive regions, flow occurs only in the active region (making the inactive region simply bypassed), and the active region displays fractal properties (Liu et al., 2005). The relative portion of the active region is dynamic and expressed as a power function of soil effective saturation, $S_{e}^{*}$, as follows:

$$
f=\left(S_{e}^{*}\right)^{\gamma}
$$

where $f$ is the portion of the active region; $\gamma$ is the ARM parameter, which is the key parameter used for describing the fractal characteristics of the preferential flow and transport properties; and $S_{e}^{*}$ is the average active water saturation for the whole flow region, defined as:

$$
S_{e}^{*}=\frac{V_{a w}}{V_{t f}}
$$

where $V_{a t}$ is the total water volume (excluding the volume corresponding to the residual water content) in the active region, $V_{t f}$ is the pore volume (excluding the volume corresponding to the residual water content) of the whole flow region (including both the active region and inactive region). Eq. (1) is the key relation in the ARM. In this study, we evaluate the ARM by examining (a) whether Eq. (1) satisfactorily represents the relevant field observations and (b) whether the parameter $\gamma$ is approximately constant with different test conditions for a given test site. We 
also investigate how the parameter $\gamma$ changes with test scale. In general, we expect it not to be a strong function of scale, because it is determined by the fractal dimension of flow pattern, and the fractal dimension is a constant at different scales for a fractal pattern.

From the definition of $f, S_{e}^{*}$ can be calculated by

$$
S_{e}^{*}=f S_{a}
$$

where $S_{a}$ is the effective water saturation within the active region, defined as

$$
S_{a}=\frac{V_{a w}}{V_{a f}}
$$

where $V_{a f}$ is the pore volume (excluding the residual pore volume) of the active region. $S_{a}$ is calculated by the following equation

$$
S_{a}=\frac{\theta_{a}-\theta_{r}}{\theta_{s}-\theta_{r}}
$$

where $\theta_{a}$ is the water content in the active region, $\theta_{s}$ is the saturated water content, and $\theta_{r}$ is the residual water content.

To evaluate the ARM, we need to determine relations between field observations and the ARM parameter $\gamma$ in Eq.(1). In the ARM, the parameter $\gamma$ is assumed to be a positive constant (between zero and one), depending on soil properties, and is used for characterizing the heterogeneous water flow properties (Liu et al., 2005). 


\subsection{The random cascade model and determination of its parameter values}

As previously discussed in Section 1, to better understand flow patterns in soil and some physical insights of the ARM, it is necessary to investigate the relations between $\gamma$ and other parameters used in the discrete approaches to describe heterogeneous flow patterns. Since both the ARM and the random cascade model were developed based on fractal behavior of unsaturated flow pattern (or flow heterogeneity), we also compared parameter values determined from both the ARM and the cascade model using field observations. Especially, we are interested in whether some intrinsic relation exists between these parameters.

The random cascade model was discussed in detail by Olsson et al., (2002); Schmitt, (2003) and Wang et al., (2006). For convenience, we briefly review it here. The infiltration depth was expressed as:

$$
Z(x, y)=Z_{A} \varepsilon(x, y)
$$

where $Z(x, y)$ is the infiltration depth at a location with coordinates $(x, y)$ in the horizontal plane, $Z_{A}$ is the mean infiltration depth, and $\varepsilon(x, y)$ is the random cascade function. The construction of a random cascade begins with the mean value of the infiltration depth over a two-dimensional $(d=2)$ bounded region. The region is successively divided into $b$ equal sub-parts $\left(b=2^{d}=4\right)$ at each step. During each subdivision, the infiltration depth obtained at each cascade from the previous step is 
distributed into the $b$ subdivisions according to a set of cascade generators $W$. All these random variables are assumed to be positive and independently distributed with the property $E\langle W\rangle=1$, where $E\langle\cdot\rangle$ denotes expectation. A field of infiltration depth, $\varepsilon(x, y)$ at the smallest scale $l_{0}$, is given by the cascade generator $W_{i}(x, y)$ as:

$$
\varepsilon(x, y)=\prod_{i=1}^{n} W_{i}(x, y)
$$

Assuming the Gaussian distribution, $W_{i}(x, y)=\exp [g]$ is used, where $g$ is a Gaussian random variable of mean $G$ and variance $\sigma^{2}$. The finial form of the cascade generators $W_{i}(x, y)$ is given as:

$$
W_{i}(x, y)=\exp \left[\sigma g_{0}-\frac{\sigma^{2}}{2}\right]
$$

Stained coverage was used to estimate $Z_{A}$ and $\sigma$. With a chosen set of $Z_{\mathrm{A}}$ and $\sigma, W$ was calculated using Eq.(8) and then infiltration depth distribution was generated using Eq (6); the generated coverage was compared with the experimental results, and the correlation coefficient between simulated and measured coverage was calculated. By repeating the procedure with different sets of $Z_{A}$ and $\sigma$, the values of $Z_{A}$ and $\sigma$ resulting in the highest correlation coefficient were considered with the final estimated parameter. The parameter $\sigma$ stands for an index to evaluate the heterogeneity of soil structure and plays a key role in determing preferential flow patterns. The $\sigma$ value increases as flow patterns become more heterogeneous (Wang 
et al., 2006).

\section{RESULTS AND DISCUSSION}

\subsection{Heterogeneous flow patterns}

Fig. 2 shows the coverage of active region (the stained region) versus depth for the plots. Binary images of typical flow patterns for the four $100 \times 100 \mathrm{~cm}^{2}$ plots at Site II are presented in Fig. 3. The differences in flow patterns among these four plots are obvious. For the total infiltrating water depths of $20 \mathrm{~mm}$ and $40 \mathrm{~mm}$, relatively homogeneous flow patterns are observed only in the upper $5 \mathrm{~cm}$ soil; then, the flow becomes preferential. The stained coverage decreases sharply with depth; further down the profiles, the stained flow paths gradually disappears. For the total infiltrating-water depth of $60 \mathrm{~mm}$, two different layers are detected in the flow patterns, showing the transition of the homogeneous infiltration zone to the preferential flow zone. Almost complete stained coverage, with few unstained spots, is observed in the top $10 \sim 20 \mathrm{~cm}$. In the soil below $20 \mathrm{~cm}$, flow changs from homogeneous infiltration to preferential flow characterized by many smaller fingers. For the total infiltrating-water depth of $80 \mathrm{~mm}$, the flow patterns are much more homogeneous than other cases, with a much larger infiltration depth and few unstained region spots in the upper layer. 
Stained patterns in vertical sections of the $100 \times 200 \mathrm{~cm}^{2}$ plot (Plot 12) and the $100 \times 400 \mathrm{~cm}^{2}$ plot (Plot 13) are shown in Figs.4 and 5, respectively. Compared with the flow patterns shown in Fig. 3(b), flow patterns in these two figures become more complex as the measurement scale increases. The stained patterns represented in Fig.3 (b) are quite simple and dominated by the narrow stain shapes, whereas the stained patterns shown in Figs.4 and 5 showed a broader distribution of shapes.

The stained flow patterns in the horizontal section at two replication plots with a total infiltrating-water depth of $40 \mathrm{~mm}$ (Plot 3 and plot 4) are shown in Fig. 6. According to these figures, stained flow patterns of the two replicates are significantly different from each other even under the same irrigation conditions.

Table 3 lists the maximum infiltration depth and the depth where $50 \%$ of soil was stained (50\% coverage depth). Averaged initial soil saturation was listed in Table 2, labeled as "Isa", for the two plots with same experimental treatment. A smaller ratio for the maximum depth and 50\% coverage depth was observed for the plots with relative higher initial saturation.

All these observations indicate that characteristics of flow patterns are dynamic and depend on water flow conditions. This is consistent with the general idea of the ARM that the relative portion of the active region is not fixed, but a function of water saturation in that region. It may be useful to emphasize that the ARM was not 
developed for describing the details of flow patterns, but for describing macroscopic (horizontally averaged [in this study]) flow behavior. Therefore, the ARM is evaluated within such a context.

\subsection{Relationship among $\gamma$, initial water content and total infiltrating-water depth}

In the active region, all the water, excluding residual water content, is assumed to be mobile. Therefore, experiments should ideally be carried out under dry soil conditions (initial soil saturation near the residual point). However, under field conditions, it is virtually impossible to carry out experiments under such conditions. To analyze the test data, we assumed that before test, all the water in the soil is immobile, although the water content is higher than the residual water content. This can be partially justified, considering that water flows much more quickly in the active (stained) region. Therefore, soil water in the "inactive" region with initial water content higher than the residual content can be approximately considered immobile at least during testing period.

Fig. 7(a)-(c) show the soil water content before and after infiltration tests for the plots located at Site I, with total infiltration-water depths of $20 \mathrm{~mm}$ (Plot 1 and Plot 2), 40 mm (Plot 3 and Plot 4), and $60 \mathrm{~mm}$ (Plot 5 and Plot 6), respectively. Although the 
initial soil water content of the plots are significantly different, vertical distributions of horizontally averaged soil water saturation in the stained regions (active regions) show similar patterns. In the upper $0 \sim 0.4 \mathrm{Z}_{\max }$ layer $\left(\mathrm{Z}_{\max }\right.$ is the maximum infiltration depth), the soil water saturation in the stained region is about 0.85 to 0.90 . Saturation in this layer was mainly affected by soil texture and porosity. In the middle 0.4 0.7Z $\mathrm{Z}_{\max }$ layer, almost the same saturation was observed, although the initial water content varies distinctly. However, compared with the upper layer, the soil water saturation decreases with depth, instead of keeping at a constant value. In the bottom layer $\left(0.7 \sim 1.0 \mathrm{Z}_{\max }\right)$, the soil water saturation decreased to the initial saturation. In addition, a further sharp decrease in the soil water saturation was observed in the plots with relatively lower initial saturation.

The relation between effective saturation and stained coverage for each plot is presented in Fig. 8. The fitted $\gamma$ values (from Eq.(1)) are listed in Table 3. In general, Eq.(1) can fit the test results reasonably well for a full range of water saturations under different test conditions (Fig. 8). In the other words, the key preferential flow feature can be captured with the ARM. The fitted $\gamma$ values are close for different initial water contents and total infiltrating water depths for a given test site (Table 3 and Fig. 9), which is consistent with an ARM assumption that the parameter $\gamma$ is approximately constant for a given site (Liu et al. 2005). 


\subsection{Relationship among model parameters ( $\gamma$ and $\sigma$ ), flow pattern and}

\section{measurement scale}

For each plot, dye-stained patterns are also analyzed using the random cascade model.

The fitted values for parameters $\mathrm{Z}_{\mathrm{A}}$ and $\sigma$ are listed in Table 3. A comparison between $\gamma$ and $\sigma$ is shown in Fig.9 (a) and (b) for the plots at Sites I and II, respectively.

As shown in Table 3 and Fig. 2 for the two replicates with the same experimental treatments (Plot 1 and Plot 2; Plot 3 and Plot 4; Plot 5 and Plot 6), water penetrated into deeper layers for the plots with higher initial water saturation. Furthermore, the $\sigma$ values for those plots with higher initial saturation are lower than the replicates with lower initial saturation. Evidently, initial soil saturation plays an important role in the formation of preferential flow path, which is in accordance with the findings of Diment and Watson (1985).

As indicated by Fig. 9, the degree of flow-pattern heterogeneity (characterized by $\sigma$ ) increases initially, and then decreases as more water infiltrates into soil. The flow patterns of the plots with total infiltrating-water depths of $40 \mathrm{~mm}$ and $60 \mathrm{~mm}$ are most heterogeneous (characterized by the largest $\sigma$ values in Fig. 9) for the $100 \times 100 \mathrm{~cm}^{2}$ scale plots at both Sites I and II. Fig. 9 also shows that $\gamma$ and $\sigma$ 
follow the similar trend, although variations in $\gamma$ are very small.

As shown in Figs. 2 and.3, for the plots with a $100 \times 100 \mathrm{~cm}^{2}$ measurement scale, flow patterns vary with top boundary condition. As the total infiltrating-water depth increased from $20 \mathrm{~mm}$ to $80 \mathrm{~mm}$ for the $100 \times 100 \mathrm{~cm}^{2}$ plots, the corresponding flow pattern changed form few preferential flow paths associated with a relatively small degree of stained coverage and a small infiltration depth, to a pattern characterized by a higher stained coverage and a larger infiltration depth, and finally to a relatively homogeneous flow pattern with few unstained area and a much larger infiltration depth. Whereas, for the plots with different measurement scales (or plot sizes), flow patterns also vary significantly and become more complex for a larger scale. For the Plot 9, Plot 12, and Plot 13, the plots with same infiltrating-water depths and different measurement scales at Site II, the corresponding $\gamma$ (and $\sigma$ ) values are not significantly different.

These results (Fig. 9) seem to indicate that there exists a relationship between $\gamma$ and $\sigma$. This is not too surprising, considering that they are all associated with fractal patterns. As previously indicated, the ARM was developed for describing macroscopic (or horizontally averaged [in this study]) flow behavior in natural unsaturated soils, rather than detailed flow patterns as those shown in Figs. 3-6. Given the mathematical simplicity of the ARM and the complexity of those flow patterns, 
the consistency between the ARM and the observations is remarkable.

As previously discussed, the parameter $\gamma$ (and $\sigma$ ) does not change significantly with measurement scale. This is consistent with an intuitive expectation that the parameter $\gamma$ is not a strong function of scale, because it determined by the fractal dimension of flow pattern and the fractal dimension is a constant at different scales for a fractal pattern. This finding is of interest for practice, because it implies that estimated values for $\gamma$ from a relatively small scale can be directly used for large-scale problems.

\section{SUMMARY AND CONCLUSIONS}

At two sites, 13 plots with various boundary conditions and measurement scales were established. Preferential flow patterns were investigated using iodine-starch staining. Test results provide useful insights into flow patterns in unsaturated soils. They show that flow pattern depends on the top boundary condition. As the total infiltrating-water depth increased from $20 \mathrm{~mm}$ to $80 \mathrm{~mm}$ for the $100 \times 100 \mathrm{~cm}^{2}$ plots, the corresponding flow pattern changed form few preferential flow paths associated with a relatively small degree of stained coverage and a small infiltration depth, to a pattern characterized by a higher stained coverage and a larger infiltration depth, and finally to a relatively homogeneous flow pattern with few unstained area and a much 
larger infiltration depth. Flow patterns are also a function of measurement scale. As measurement scale increased, flow patterns become more complex. Initial soil water saturation also plays an important role in determining soil water distribution and heterogeneous flow patterns. Under the same experimental treatment, for the plots with relative higher initial soil water saturation, the flow patterns were less heterogeneous. Similar redistributions of infiltration in the stained region (active region) at various depths were observed. In the upper layer $\left(0 \sim 0.4 \mathrm{Z}_{\max }\right)$, saturation is about $0.85 \sim 0.95$. In the middle layer $\left(0.4 \sim 0.7 \mathrm{Z}_{\max }\right)$, saturation decreases with depth; however, initial soil water saturation has little influence on the saturation distributions after infiltration. In the bottom layer $\left(0.7 \sim 1.0 \mathrm{Z}_{\max }\right)$, soil water saturation decreases to the initial soil saturation, and greater decrease in soil water saturation was observed in the plots with relatively lower initial saturation.

The test results provide a valuable data set for evaluating the ARM. It was found that the water flow patterns under different test conditions are satisfactorily described by the ARM relation (Eq.(1)), supporting the validity of the ARM. While details of flow patterns (or flow heterogeneity) are obviously dependent on specific test conditions, our analysis results show that the fitted values for the ARM parameter is not sensitive to the considered test conditions including the initial water content and top boundary condition for a given test site. This is consistent with an ARM 
assumption that the parameter $\gamma$ is approximately constant for a given site.

We also examine relations among the ARM parameter $\gamma$, the random cascade model parameter $\sigma$, and measurement scale. For the plots at the experimental scale of $100 \times 100 \mathrm{~cm}^{2}$ at both Sites I and II, the values of parameter $\gamma$ (and $\sigma$ ) increase initially and then decrease with total amount of infiltrating water (ranging from 20 to $80 \mathrm{~mm}$ ), indicating that the degree of flow heterogeneity increases first and then decreases. However, variations in ARM parameter $\gamma$ with the amount of infiltrating water are small (Fig. 9) and are considered to be negligible in practice. For the plots with different measurement scales at Site II, as measurement scale increases, flow patterns became more complex and heterogeneous. However, the parameter $\gamma$ for these plots were not significantly different, demonstrating that the parameter may not be scale dependent. This may be because the parameter $\gamma$ is determined by the fractal dimension (of flow pattern) that remains a constant at different scales for a given fractal pattern.

\section{Acknowledgements}

This research was financially supported in part by grants of the National Basic Research Program of China (No. 2006CB403404), and the National Science Foundation of China (Nos. 50528910, 50579079, and 50779080). Dr. Liu was also supported by United States Department of Energy Contract No. DE-AC02-05CH11231. 


\section{REFERENCES}

Behrens, H., 1982. New insights into the chemical behaviour of radioiodine in aquatic environments. Environmental migration of long-lived radionuclides. Proc. of an Int. Symp. on Migration in the Terrestrial Environment of Long-Lived Radionuclides from the Nuclear Fuel Cycle, Knoxville, TN. 21 - 31-July 1981, $27-40$.

de Rooij, G.H., 2000. Modeling fingered flow of water in soils owing to wetting front instability: a review. J. Hydrol. 231-232, 277- 294.

Diment G A, Watson K K. 1985. Stability analysis of water movement in unsaturated porous media. 3. Experimental studies. Water Resources Research. 21:979 - 984.

Doughty, C., 1999. Investigation of conceptual and numerical approaches for evaluating moisture, gas, chemical, and heat transport in fractured unsaturated rock. J. Contam. Hydrol. 38, 69- 106.

Flury, M., Flu“ hler, H., 1995. Modeling solute leaching in soils by diffusion-limited aggregation: basic concepts and applications to conservative solutes. Water Resour. Res. 31, 2443- 2452.

Flury, M., and Flühler, H., 1995. Modeling solute leaching in soils by diffusion-limited aggregation: Basic concepts and applications to conservative solutes. Water Resour. Res. 31, 2443-2452 
Forrer, I., Parrita, A., Kasteel, R., Fluhler, H., Luca, D., 2000. Quantifying dye tracers in soil profiles by image processing. Eur. J. Soil. Sci. 51, 313-322.

Glass, R.J., Steenhuis, T.S., Parlarge, J.Y., 1988. Wetting front instability as a rapid and far-reaching hydrologic process in the vadose zone. J. Contam. Hydrol. 3, 207-226.

Hangen, E., H.H.Gerke, W.Schaaf, anf R.F. Huttl. 2004. Flow path visualization in a lignitic mine soil using iodine-starch staining. Geodema, 120:121-135

Ketelsen, H., Meyer-Windel, S., 1999. Adsorption of brilliant blue FCF by soils. Geoderma. 90, 131-145.

Kasteel R, Vogel H J, Roth K. 2002. Effect of non-linear adsorption on the transport behavior of Brilliant blue in a field soil. European Journal of Soil Science. $53: 231 \sim 240$

Larsson M H, Jarvis N J, Torstensson G, et al., 1999. Quantifying the impact of preferential flow on solute transport to tile drains in a sandy field soil. Journal of Hydrology. 215:116 134 .

Liu, H.H., C. Doughty, and G.S. Bodvarsson. 1998. An active fracture model for unsaturated flow and transport in fractured rocks. Water Resour. Res. $34: 2633-2646$.

Liu, H.H., G. Zhang., Bodvarsson. G.S. 2003. The active fracture model: Its relation 
to fractal flow patterns and an evaluation using field observations. Vadose Zone J. 2: 59-269.

Liu, H.H., R. Zhang, and G. S. Bodvarsson. 2005.An active region model for capturing fractal flow patterns in unsaturated soils: Model development. J. Contam. Hydrol. 80:18-30.

Lu J., and L. Wu. 2003. Visualizing bromide and iodide water tracer in soil profiles by spray methods, J. Environ. Qual., 32:363-367.

Morris C., and Mooney S. J. 2004. A high-resolution system for the quantification of preferential flow in undisturbed soil using observations of tracers. Geoderma, 118: $133-143$.

Ohrstrom, P., Persson, M., Albergel, J., Zante, P., Nasri, S., Berndtsson, R., Olsson, J., 2002. Field-scale variation of preferential flow as indicated from dye coverage. J. Hydrol. 257, 164-173.

Ohrstrom, P., Hamed, Y., Persson, M., Berndtsson, R., 2004. Characterizing unsaturated solute transport by simultaneous use of dye and bromide. J. Hydrol. $289,23-35$.

Olsson, J., Persson, M., Albergel, J., Berndtsson, R., Zante, P., Ohrstrom, P., Nasri, S., 2002. Multiscaling analysis and random cascade modeling of dye infiltration. Water Resour. Res. 38 (11), 1263. doi:10.1029/2001WR00080. 
Persson, M., Yasuda, H., Albergel, H., Berndtsson, R., Zante, P., Nasri, S., Ohrstrom, P., 2001. Modeling plot scale dye penetration by a diffusion limited aggregation (DLA) model. J. Hydrol. 250, 98- 105.

Pruess, K., B. Faybishenko, and G.S. Bodvarsson. 1999. Alternative concepts and approaches for modeling flow and transport in thick unsaturated zones of fractured rocks. J. Contam.Hydrol. 38:281-322.

Schmitt, F.G., 2003. A causal multifractal stochastic equation and its statistical properties. Eur. Phys. J. B 34, 85-98.

Scol, Y., T.J. Kneafsey, and K. Ito. 2006. An evaluation of the active fracture concept in the modeling unsaturated flow and transport in the fractured meter-sized block of rock, Vadose Zone J., 5:1-13

Simunek, J., Jarvis, N.J., van Genuchten, M.Th., Gardenas, A., 2003. Review and comparison of models for describing non-equilibrium and preferential flow and transport in the vadose zone. J. Contam. Hydrol. 272, 14- 35.

van Dam J C, Hendrickx J H M, van Ommen H C, et al., 1990. Water and solute movement in a coarse-textured water-repellent field soil. Journal of Hydrology, $1990,120: 359 \sim 379$

van Dam, J.C., Wosten, J.H.M., Nemes, A., 1996. Unsaturated soil water movement in hysteretic and water repellent field soils. J. Hydrol. 184, 153- 173. 
van Ommen, H.C., Dekker, L.W., Dijksma, R., Hulshof, J., van der Molen, W.H., 1988. A new technique for evaluating the presence of preferential flow paths in nonstructured soils. Soil Sci. Soc. Am. J. 52, 1192- 1193.

Yasuda, H., R. Berndtsson, H. Persson, A. Bahri, K. Takuma. 2001. Characterizing preferential transport during flood irrigation of a heavy clay soil using the dye Vitasyn Blua. Geoderma, 100: 49-66.

Wang, K., R. Zhang, H.Yasuda. 2006. Characterizing heterogeneity of soil water flow by dye infiltration experiments. J. hydrol., 328:559-571.

Wang, Z., Lu, J., Wu, L., Harter, T., Jury, W.A., 2002. Visualizing preferential flow paths using ammonium carbonate and a pH indicator. Soil Sci. Soc. Am. J. 66, $347-351$.

Wang, Z., L.Wu, T.Harter, J.Lu, and W.A. Jury. 2003. A field study of unstable preferential flow during soil water redistribution. Water Resour. Res., 39(4),doi: 10,1029/2001WR000903. 
Table 1 . Soil physical and hydraulic properties

\begin{tabular}{|c|c|c|c|c|c|c|c|}
\hline \multirow{2}{*}{ Site } & \multirow{2}{*}{$\begin{array}{l}\text { depth } \\
\mathrm{cm}\end{array}$} & \multicolumn{3}{|c|}{ texture } & \multirow{2}{*}{$\begin{array}{l}\text { bulk } \\
\text { density } \\
\mathrm{g} \mathrm{cm}^{-3}\end{array}$} & \multirow{2}{*}{$\begin{array}{l}\text { porosity } \\
\%\end{array}$} & \multirow{2}{*}{$\begin{array}{c}\text { saturated hydraulic } \\
\text { conductivity } \\
\mathrm{cm} \mathrm{s}^{-1}\end{array}$} \\
\hline & & $\begin{array}{c}>50 \mu m \\
\%\end{array}$ & $\begin{array}{c}2 \sim 50 \mu m \\
\%\end{array}$ & $\begin{array}{c}<2 \mu m \\
\%\end{array}$ & & & \\
\hline \multirow{4}{*}{ I } & $0-10$ & 29.4 & 49.2 & 21.4 & 1.38 & 38.8 & $3.4 \times 10^{-4}$ \\
\hline & $10-20$ & 28.2 & 49.4 & 22.4 & 1.40 & 40.2 & $1.2 \times 10^{-4}$ \\
\hline & $20-50$ & 31.3 & 46.2 & 22.5 & 1.44 & 40.6 & $1.1 \times 10^{-4}$ \\
\hline & $50-100$ & 32.1 & 44.8 & 23.1 & 1.45 & 44.4 & $1.1 \times 10^{-4}$ \\
\hline \multirow{4}{*}{ II } & $0-10$ & 3.5 & 55.0 & 44.5 & 1.44 & 40.2 & $5.8 \times 10^{-5}$ \\
\hline & $10-20$ & 4.4 & 51.5 & 44.4 & 1.50 & 42.7 & $2.1 \times 10^{-5}$ \\
\hline & $20-50$ & 4.3 & 51.4 & 44.3 & 1.50 & 44.8 & $2.2 \times 10^{-5}$ \\
\hline & $50-100$ & 4.7 & 50.7 & 44.6 & 1.59 & 45.1 & $1.4 \times 10^{-5}$ \\
\hline
\end{tabular}

Table 2. The dye infiltration conditions of each plot

\begin{tabular}{|c|c|c|c|c|c|}
\hline \multirow{2}{*}{$\begin{array}{l}\text { Infiltration amount } \\
\qquad(\mathrm{mm})\end{array}$} & \multirow{2}{*}{\multicolumn{2}{|c|}{$\frac{\text { Loam }}{100 \times 100 \mathrm{~cm}^{2}}$}} & \multicolumn{3}{|c|}{ Clay } \\
\hline & & & $100 \times 100 \mathrm{~cm}^{2}$ & $100 \times 100 \mathrm{~cm}^{2}$ & $100 \times 100 \mathrm{~cm}^{2}$ \\
\hline 20 & Plot 1 & Plot 2 & Plot 8 & I & l \\
\hline 40 & Plot 3 & Plot 4 & Plot 9 & Plot 12 & Plot 13 \\
\hline 60 & Plot 5 & Plot 6 & Plot 10 & l & l \\
\hline 80 & Plot 7 & I & Plot 11 & / & l \\
\hline
\end{tabular}

Table 3. Parameters for describing flow heterogeneity and fractal.

\begin{tabular}{|c|c|c|c|c|c|c|c|c|}
\hline Texture & Plot & $\mathrm{Z}_{50}$ & $\mathrm{Z}_{\max }$ & $\mathrm{ISa}$ & $\mathrm{Z}_{50} / \mathrm{Z}_{\max }$ & $\mathrm{Z}_{\mathrm{A}}$ & $\sigma$ & $\gamma$ \\
\hline \multirow{5}{*}{$\begin{array}{c}\text { Loam } \\
\text { (Site I) }\end{array}$} & Plot 1 & 6.0 & 46.0 & 0.6134 & 0.13 & 6.5 & 0.302383 & 0.6181 \\
\cline { 2 - 9 } & Plot 2 & 12.0 & 41.3 & 0.7330 & 0.29 & 18.2 & 0.25057 & 0.7366 \\
\cline { 2 - 9 } & Plot 3 & 14.4 & 51.0 & 0.4840 & 0.28 & 18.8 & 0.650772 & 0.7882 \\
\cline { 2 - 9 } & Plot 4 & 11.8 & 54.8 & 0.3470 & 0.21 & 19.3 & 0.500548 & 0.7198 \\
\cline { 2 - 10 } & Plot 5 & 18.0 & 83.0 & 0.4729 & 0.21 & 18.2 & 0.401147 & 0.7208 \\
\cline { 2 - 9 } & Plot 7 & 56.8 & 114.5 & 0.5965 & 0.49 & 48.6 & 0.446059 & 0.6235 \\
\hline \multirow{5}{*}{$\begin{array}{c}\text { Clay } \\
\text { (Site II ) }\end{array}$} & Plot 8 & 9.7 & 41.7 & 0.7093 & 0.23 & 16.6 & 0.501156 & 0.7203 \\
\cline { 2 - 9 } & Plot 9 & 23.4 & 85.4 & 0.5981 & 0.27 & 26.4 & 0.607724 & 0.7442 \\
\cline { 2 - 9 } & Plot11 & 43.4 & 108.2 & 0.6387 & 0.40 & 64.4 & 0.518732 & 0.6619 \\
\cline { 2 - 9 } & Plot12 & 14.2 & 90.2 & 0.5022 & 0.16 & 22.4 & 0.658258 & 0.7469 \\
\cline { 2 - 9 } & Plot13 & 30.3 & 98.4 & 0.4747 & 0.31 & 30.4 & 0.685169 & 0.7618 \\
\hline
\end{tabular}

ISa: Average initial saturation. 


\section{Figure List}

Figure 1. Diagram of experimental set-up

Figure 2. Coverage of stained region vs. depth

Figure 3. Flow patterns of the $100 \mathrm{~cm}$ size plots at site II (Plot 8, Plot 9, Plot 10 and Plot 11.)

Figure 4. Flow patterns of the $200 \mathrm{~cm}$ size plots at site II (Plot 12)

Figure 5. Flow patterns of the $400 \mathrm{~cm}$ size plots at site II (Plot 13)

Figure 6. Comparison of stained patterns at various depths between two plots with same ponding water of 40mm (Plot 3 and Plot 4)

Figure 7. Comparison between initial soil saturation and saturation at the active region (stained region) for the plots with same experimental treatment (Plot 1 vs. Plot 2, Plot 3 vs. Plot 4, and Plot 5 vs. Plot 6 )

Figure 8. Relationships between soil saturation and coverage of stained region, which were used to fit Arm parameter $\gamma$.

Figure 9. Comparison of ARM parameter $\gamma$ with random cascade model parameter $\sigma$, both of them were related to flow heterogeneity. 


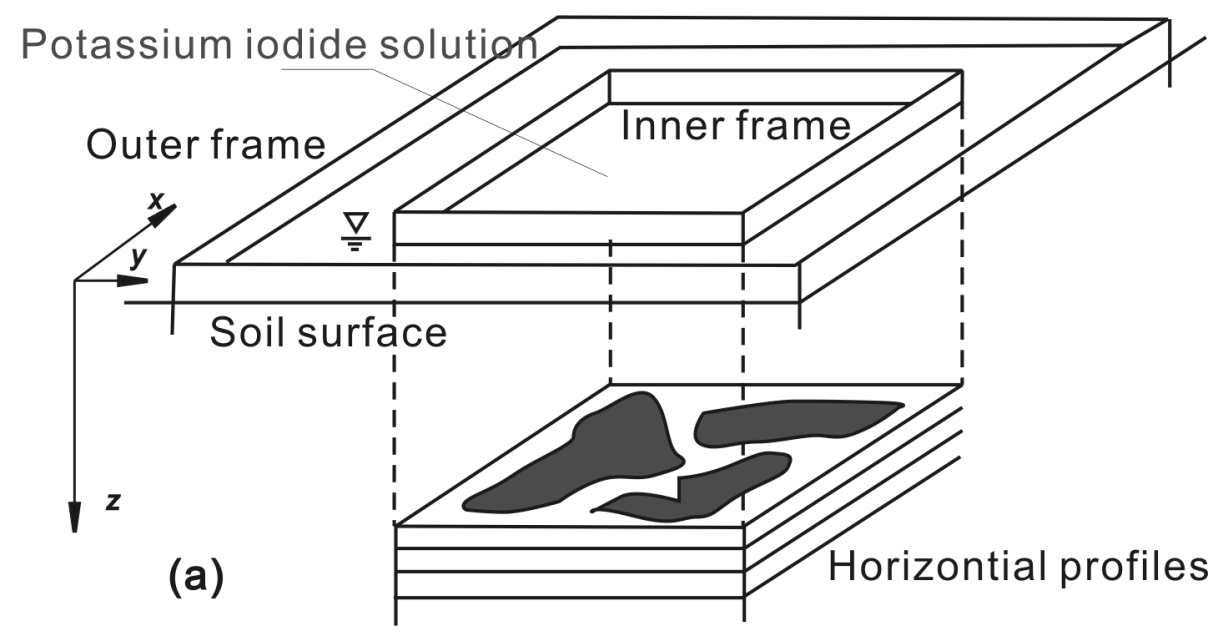

Site I

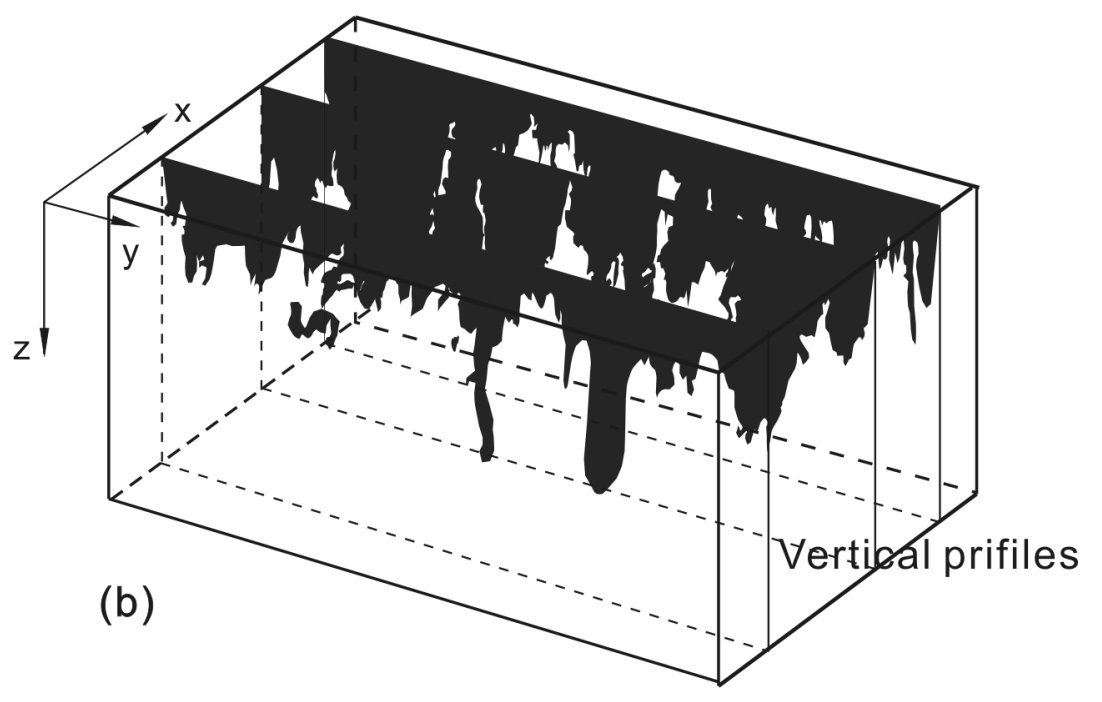

Site II

Figure 1 


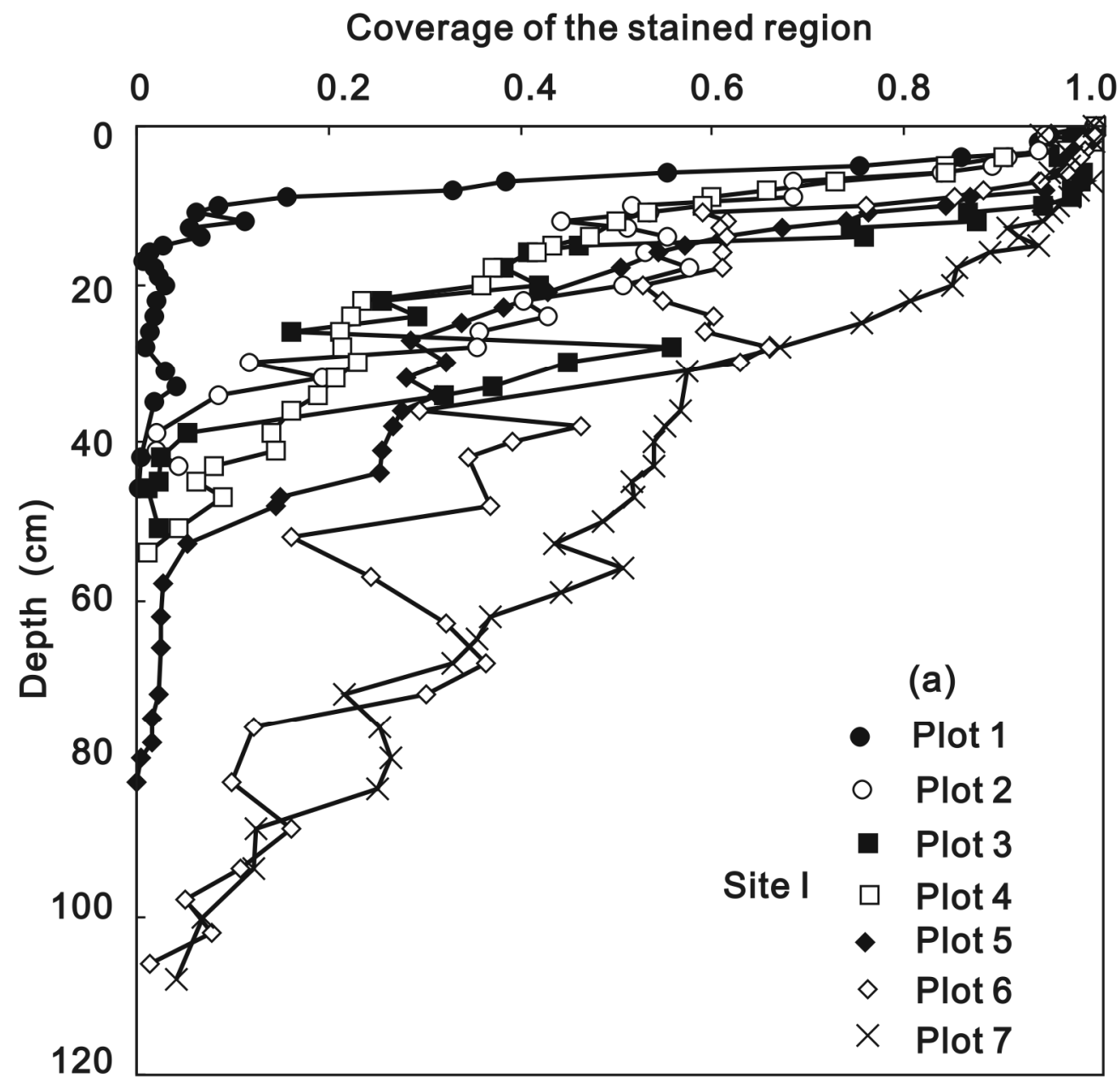




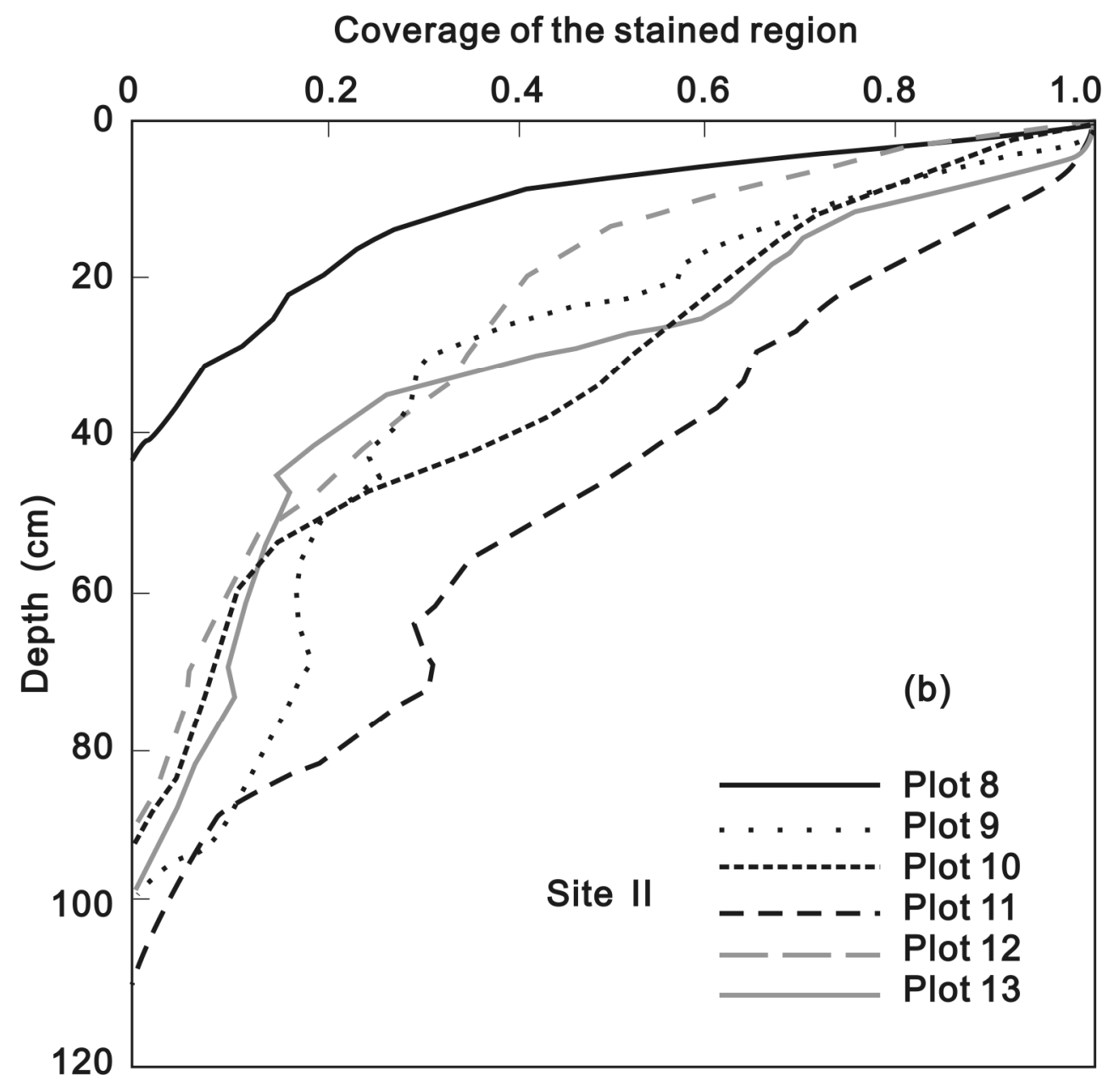

Figure 2 

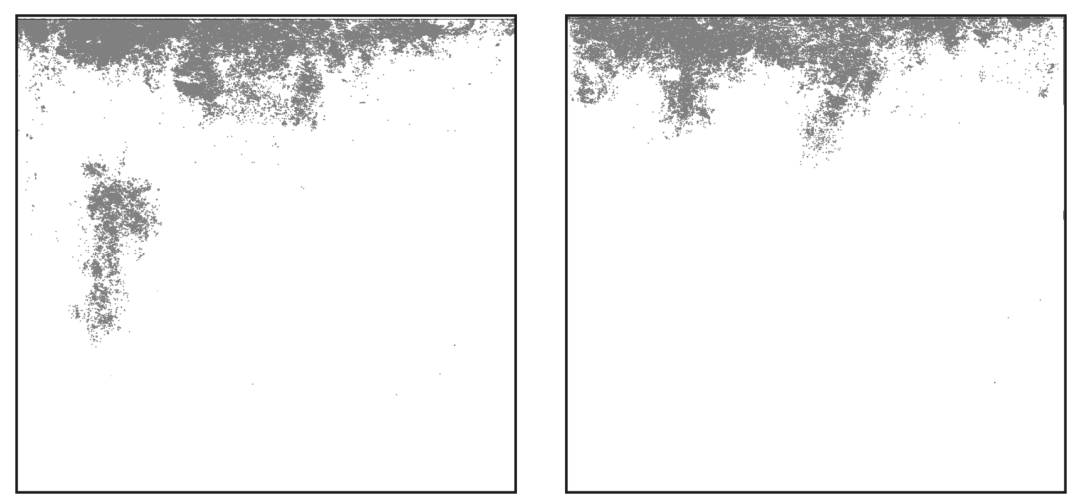

(a) Plot 8 (ponding water: $20 \mathrm{~mm}$ )
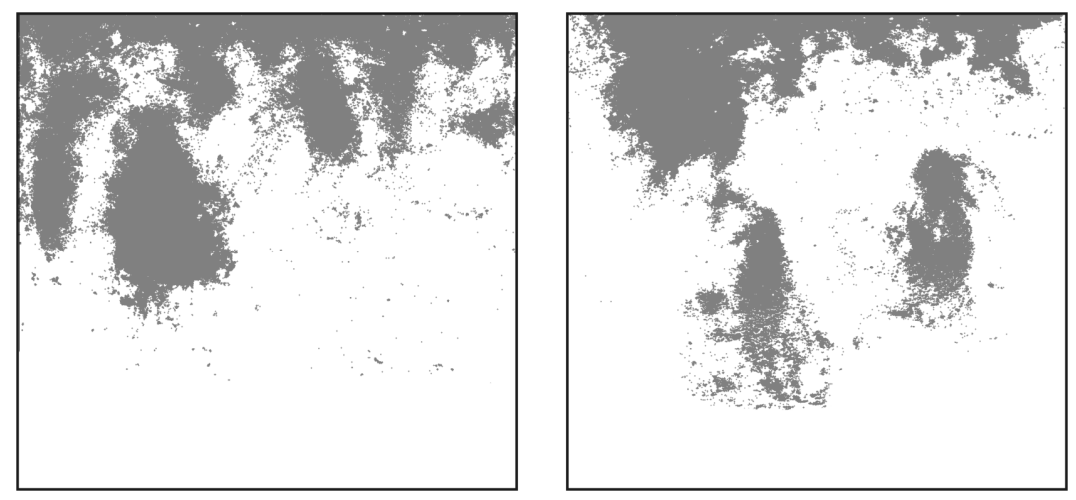

(b) Plot 9 (ponding water: 40mm)
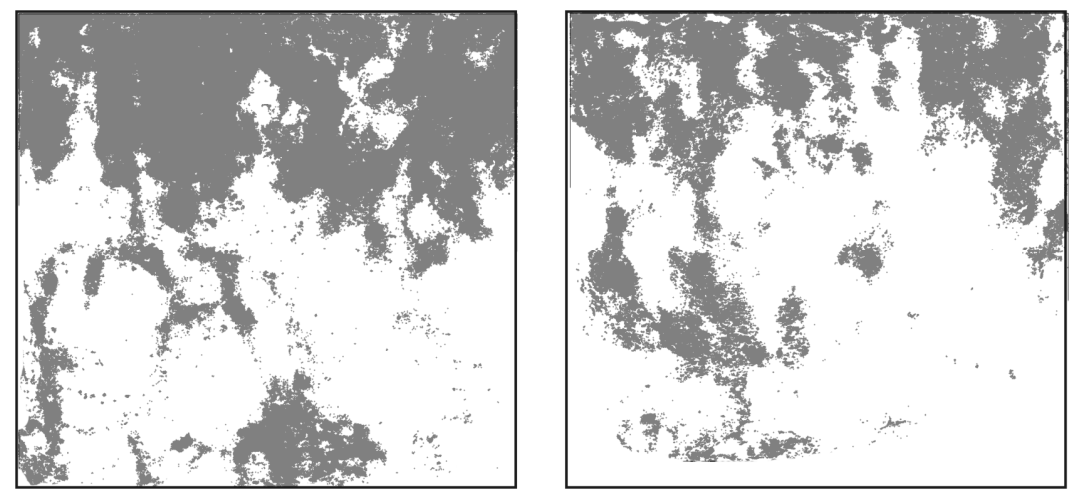

(c) Plot 10 (ponding water: 60mm) 

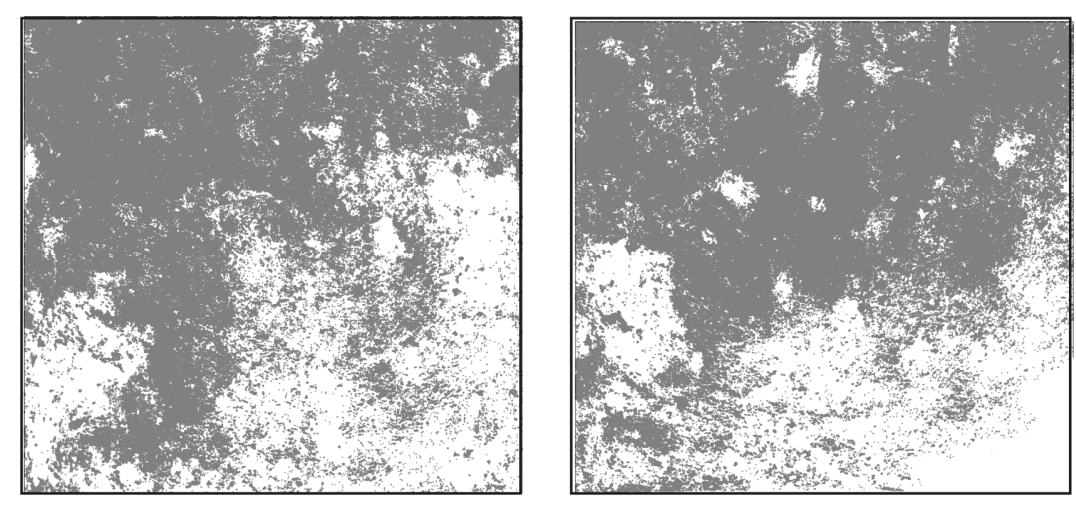

(d) Plot 11 (ponding water: $80 \mathrm{~mm}$ )

Figure 3 


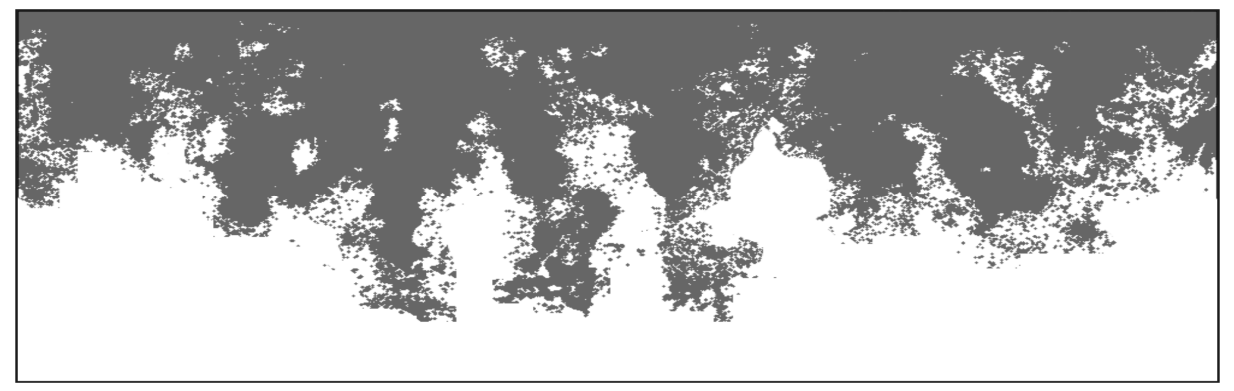

(a) Plot $12 \mathrm{x}=40 \mathrm{~cm}$

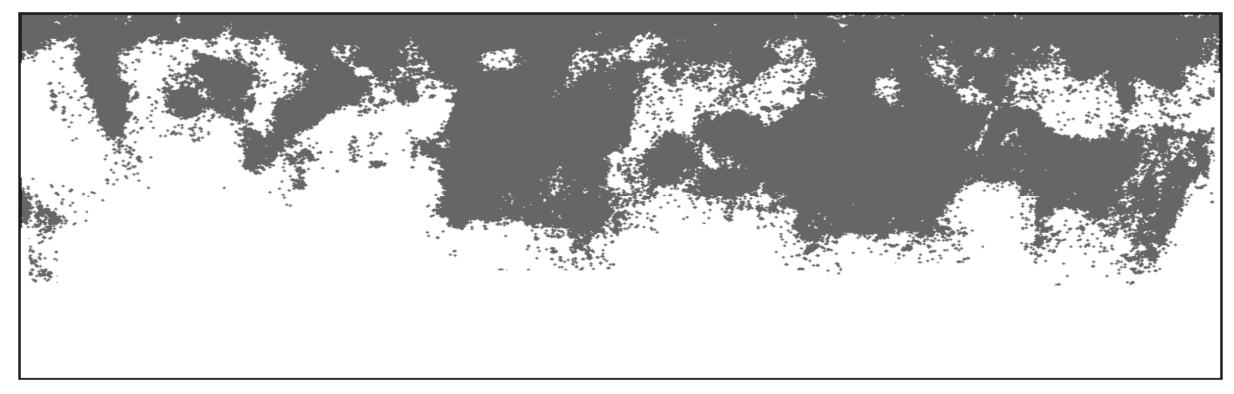

(b) $x=60 \mathrm{~cm}$

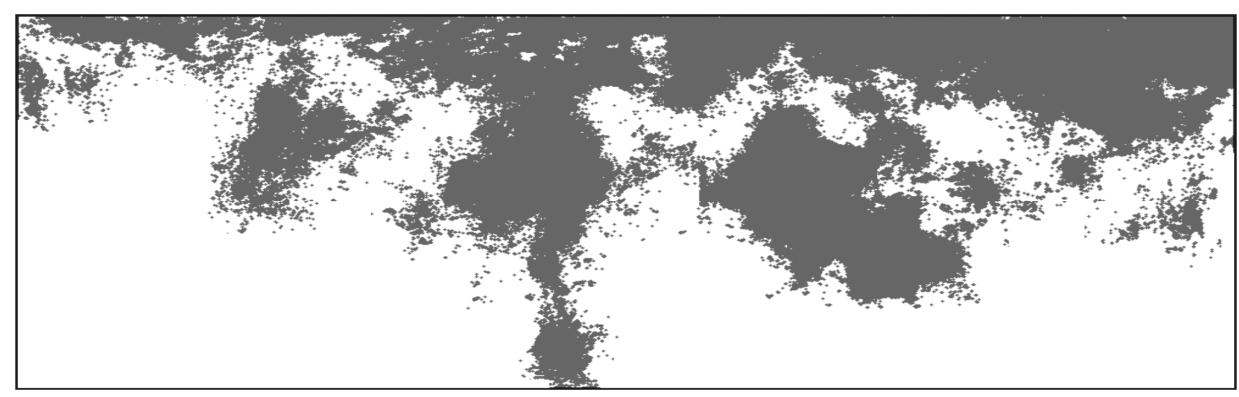

(b) $x=80 \mathrm{~cm}$

Figure 4 


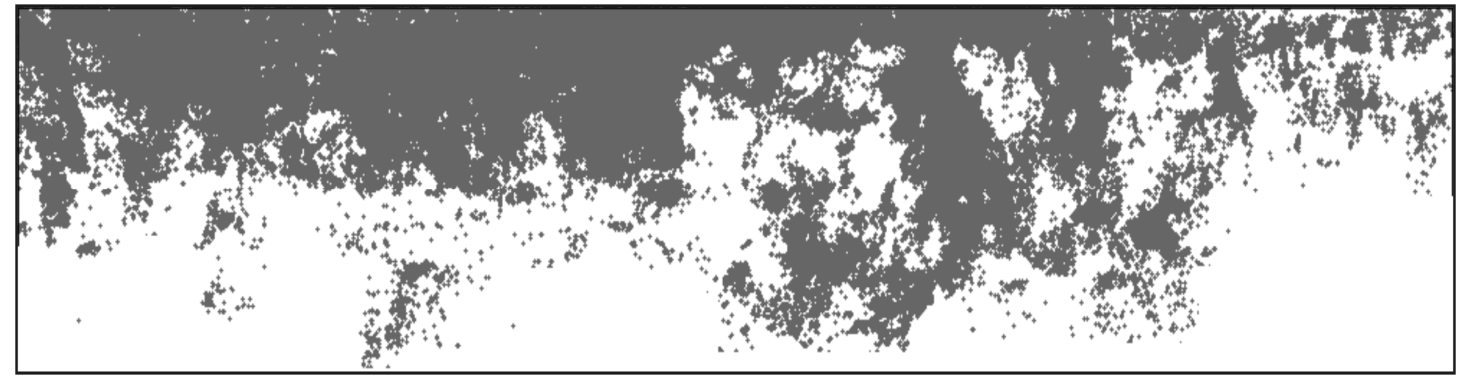

(a) Plot $13 \mathrm{x}=40 \mathrm{~cm}$

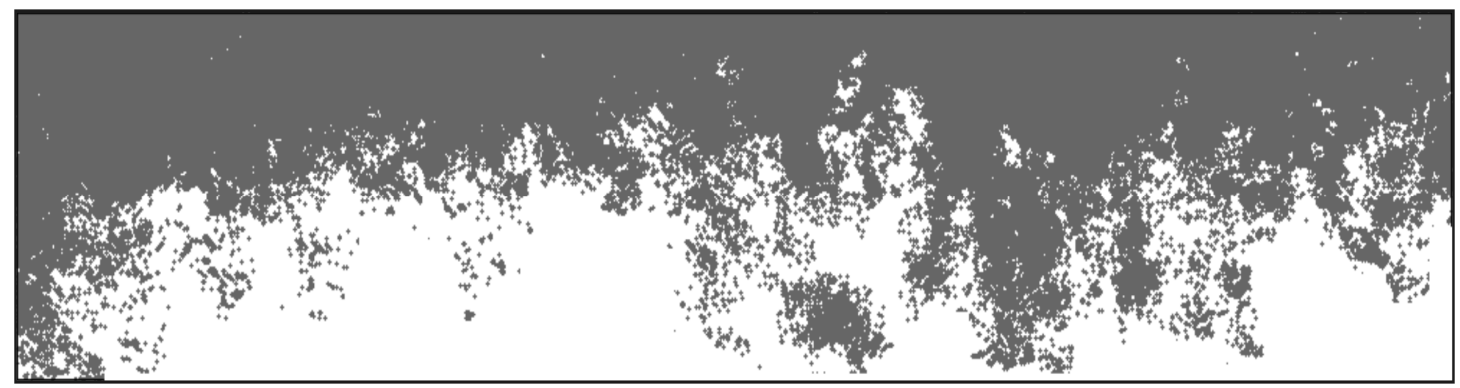

(b) Plot $13 \mathrm{x}=60 \mathrm{~cm}$

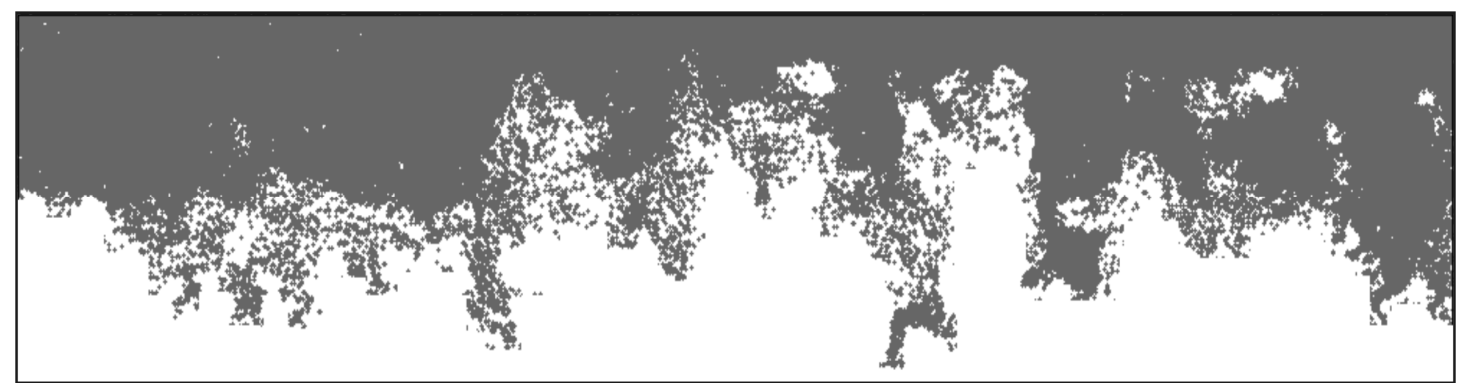

(c) Plot $13 \mathrm{x}=80 \mathrm{~cm}$

Figure 5 

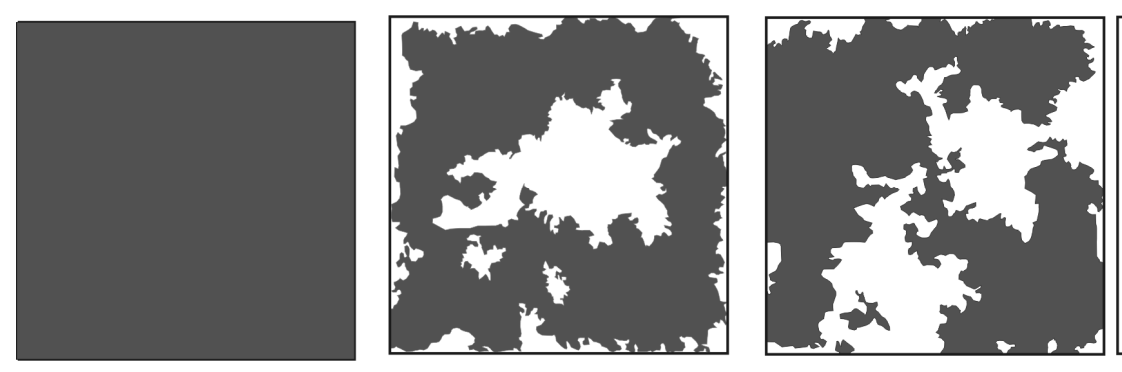

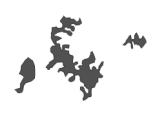

$\mathrm{z}=5 \mathrm{~cm}$

$\mathrm{z}=10 \mathrm{~cm}$

$\mathrm{z}=20 \mathrm{~cm}$

$\mathrm{z}=40 \mathrm{~cm}$

(a) Plot 3
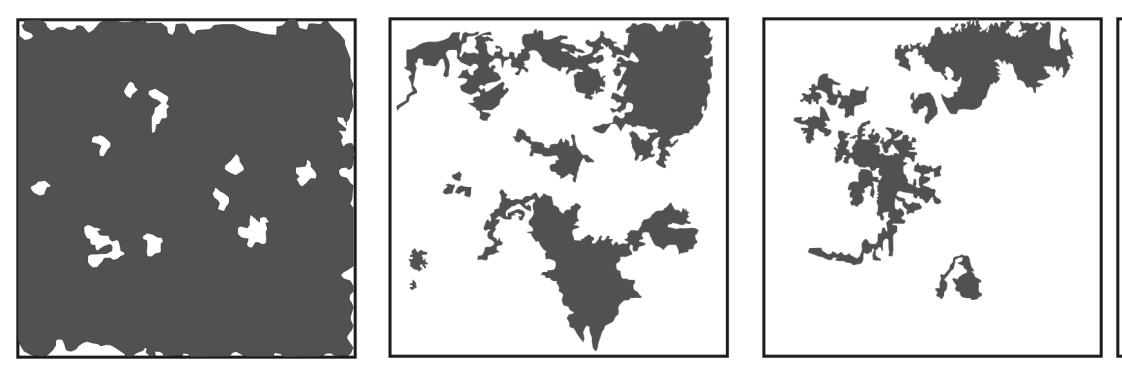

$+$

$\mathrm{z}=5 \mathrm{~cm}$

$\mathrm{z}=10 \mathrm{~cm}$

$\mathrm{z}=20 \mathrm{~cm}$

$\mathrm{z}=40 \mathrm{~cm}$

(b) Plot 4

Figure 6 

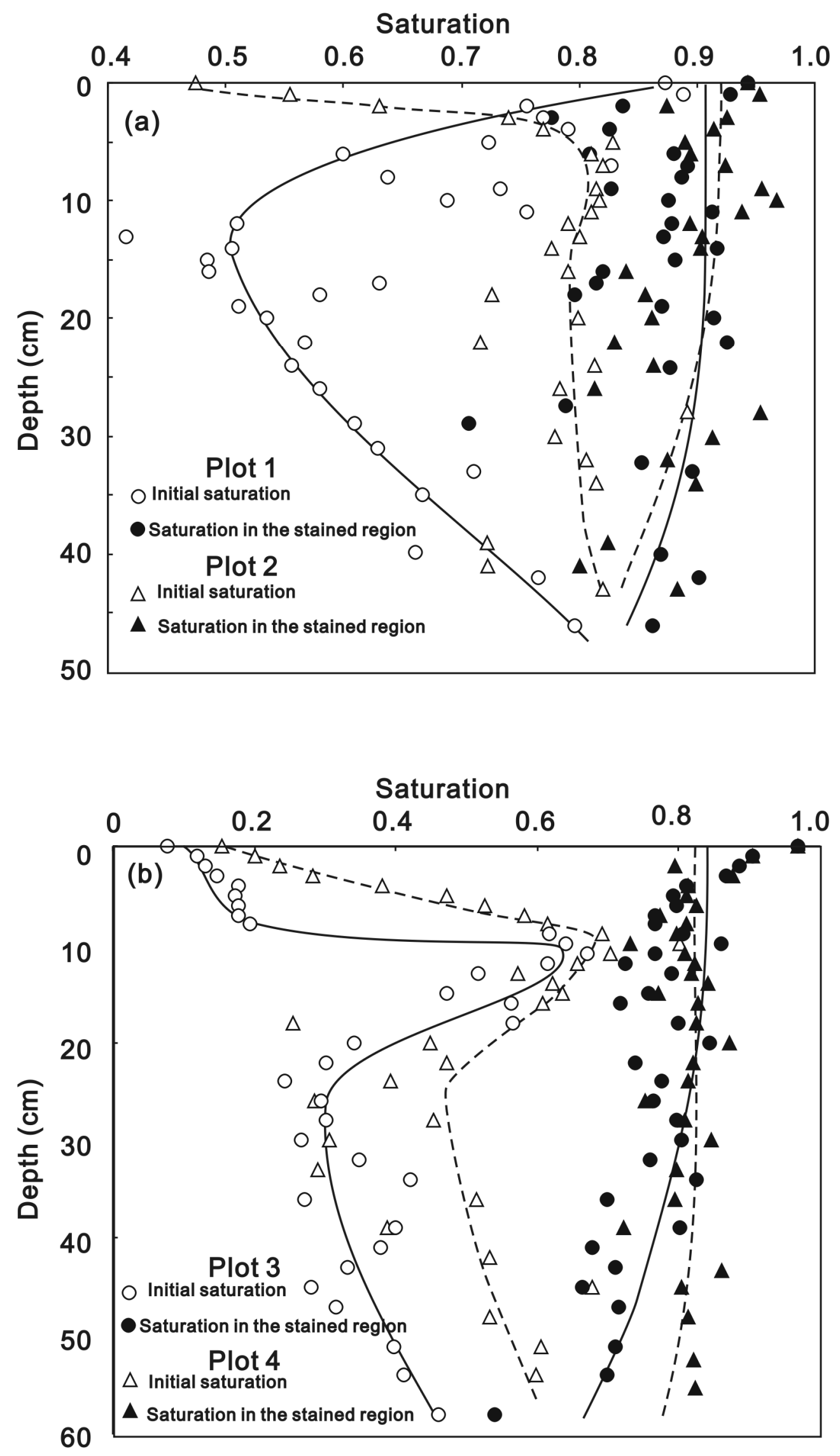


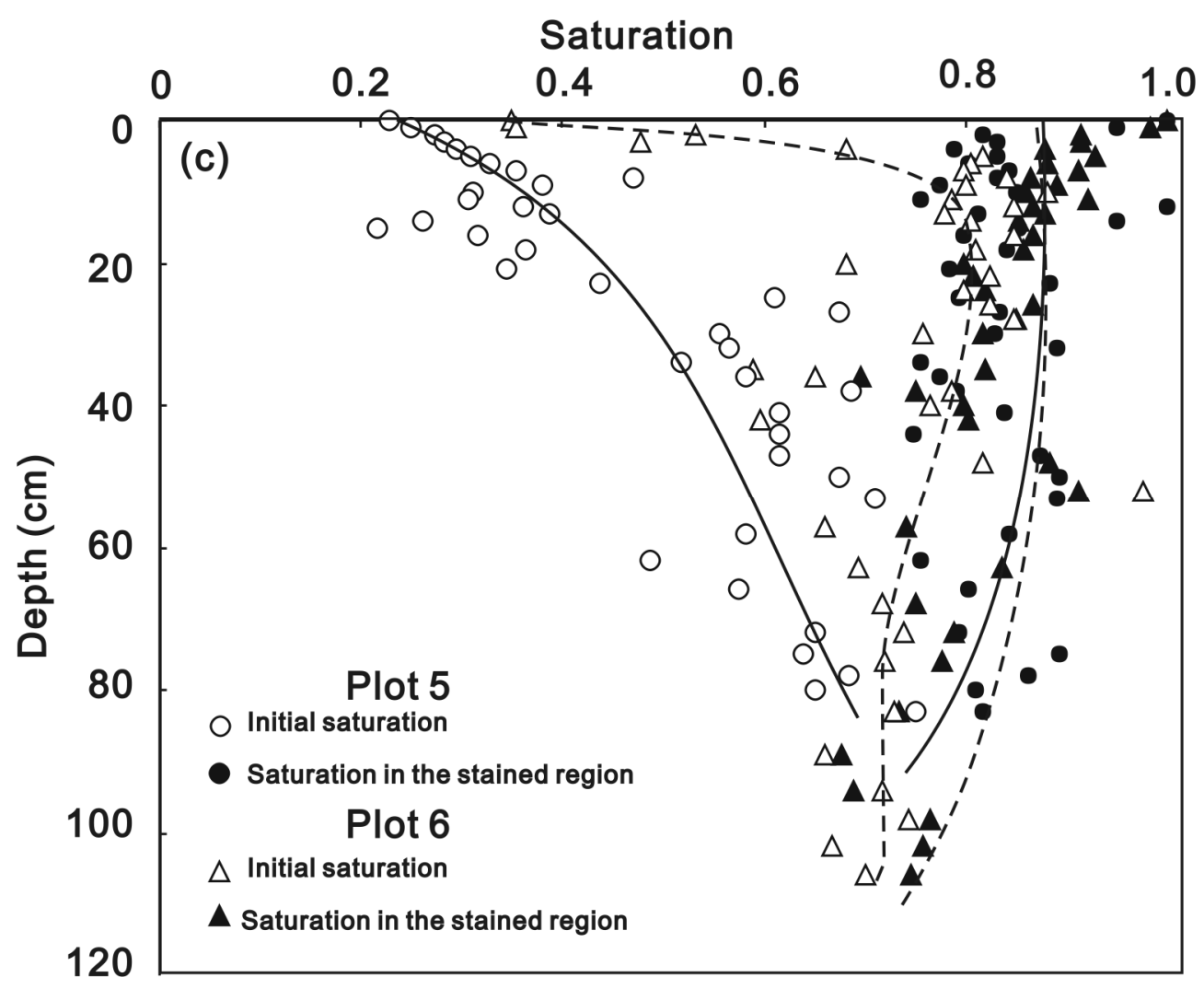

Figure 7 

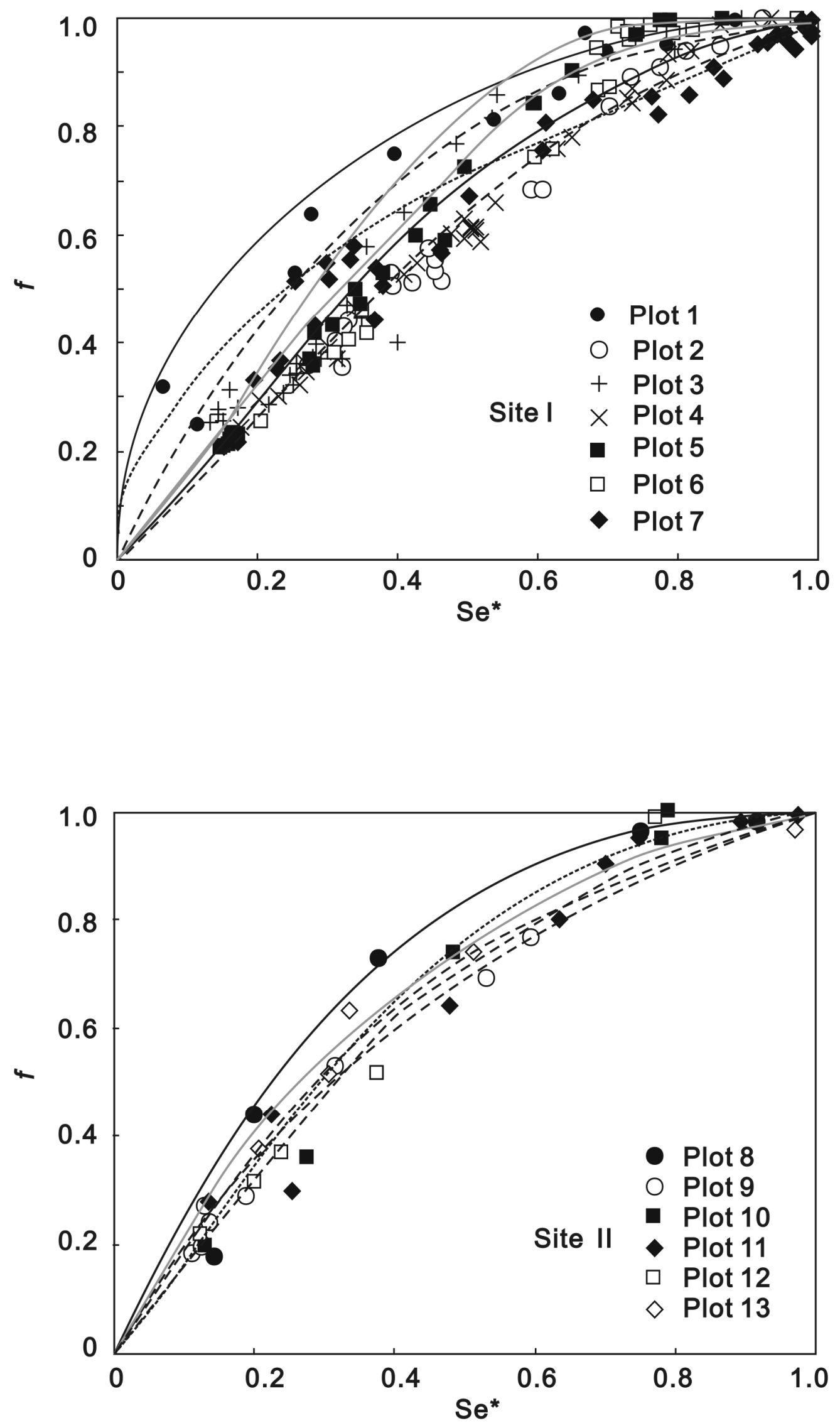

Figure 8 

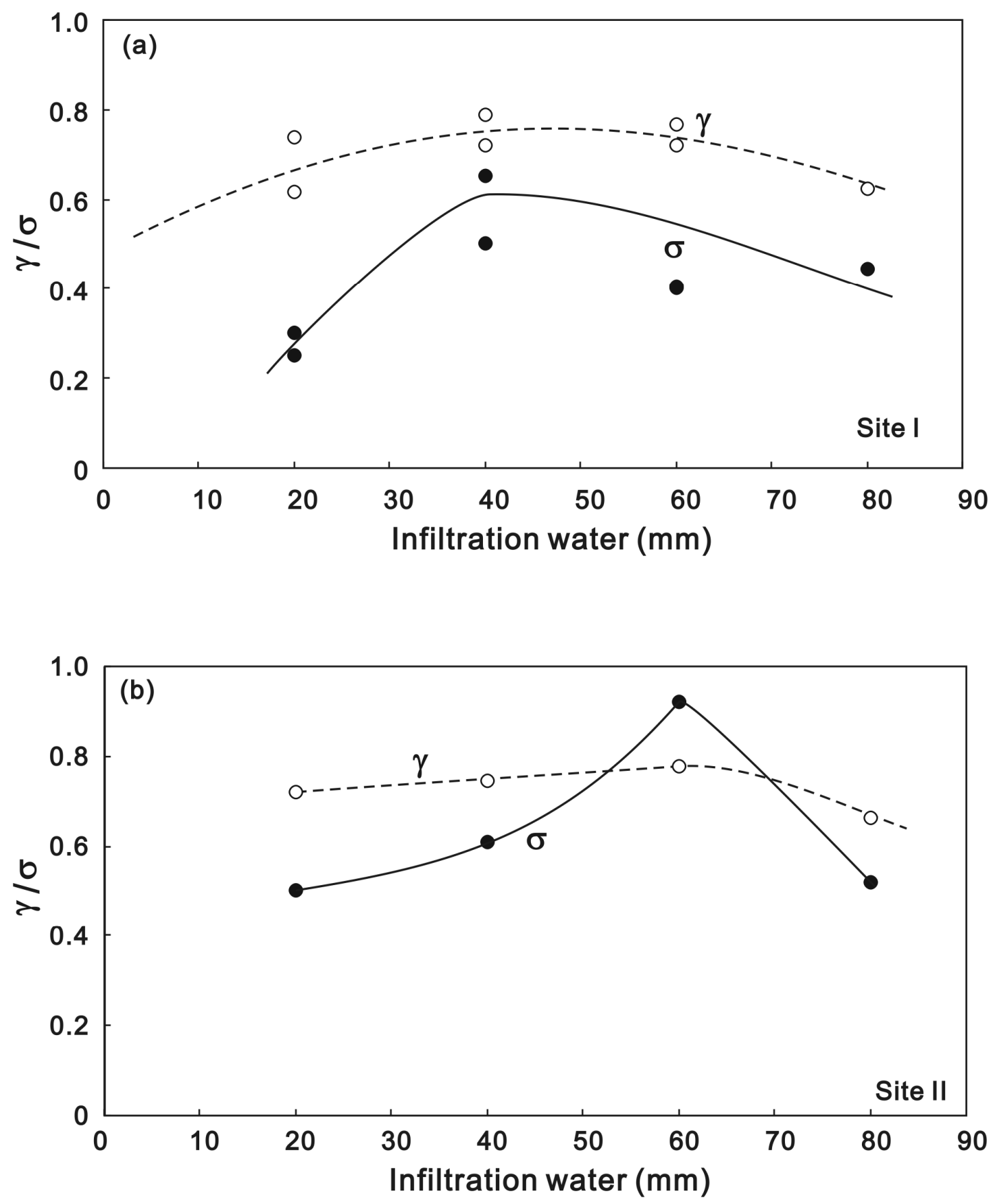

Figure 9 